\title{
Effect and Design of Microstrip Patch Antenna with Defected Ground Structure
}

\author{
Sukhamrit Pal Singh Sodhi, Priya Chabra \\ Ludhiana College of Engineering \& Technology; \\ Ludhiana
}

\begin{abstract}
Analysis of resonant behavior of microstrip patch antenna with defective ground structure has been presented in this paper. It is observed that a size reduction of $55.66 \%$ has been achieved by taking out an $h$ shaped slot from both the patch and ground plane. As a result this new antenna exhibits multiband behavior and Better resonant characteristics than its original structure the proposed antenna not only has characteristics of good radiation. But also has an advantage of low cost and small size for $\mathbf{S}$ band $\mathbf{C}$ band and $\mathrm{X}$ band wireless applications
\end{abstract}

Keywords-DGS, microstrip antenna, return loss, gain.

\section{INTRODUCTION}

There is a remarkable progress going on in wireless communication system. Increasing demand to combine different Technologies into Micro equipment has greatly increased. the demand of introducing compact emptiness . microstrip patch antenna comma because of its small size, low profile, Low building cost and ease of working with feed networks are extensive applications in wireless communication system. With Very thin profile (.01 to .05 wavelength) microstrip antennas have got great applications in military missiles Rockets, aircrafts and satellites defected ground structure is etched periodic or non periodic cascade defects in ground plane of microstrip antenna. Defect in ground plane helps in increasing capacitance and inductance. In this paper a small size microstrip antenna with slot and defective ground structure have been designed to examine the relationship between the resonant performances of these antennas.

\section{ANTENNA DESIGN \& STRUCTURE}

Fig.1 shows the basic geometry of the proposed patch antenna with slot and DGS structure.

(a)

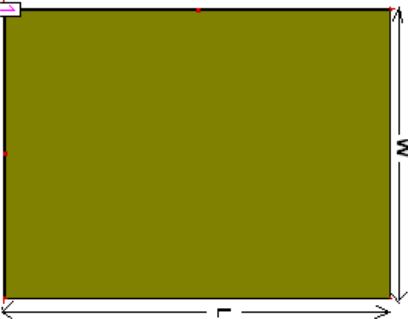

(c)

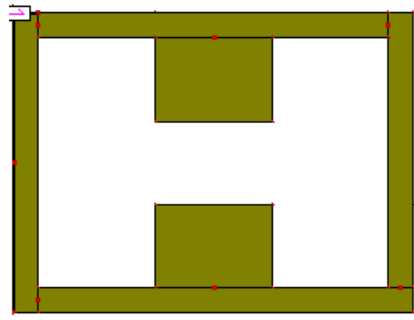

(b)

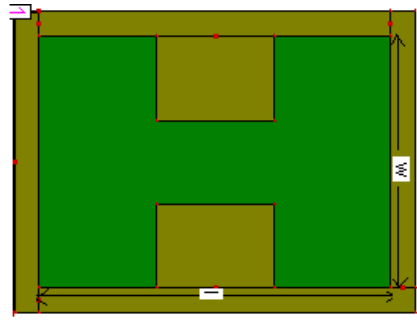

(d)

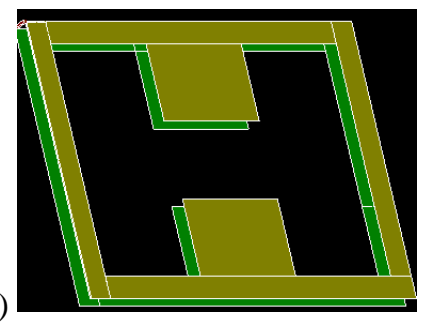

Fig.1 Geometrical Construction of the Proposed Antennas, (a) micro strip antenna (b) micro strip antenna with slot (c) micro strip antenna with slot and DGS (d) 3D view of proposed structure.

The antennas have been design with fr4 substrate having height of $1.6 \mathrm{~mm}$. The height is a small sized planar patch antenna, the that has its length, $\mathrm{L}=24 \mathrm{~mm}$ and width $\mathrm{W}=32 \mathrm{~mm}$. the presented second antenna structure has $\mathrm{H}$ shaped slot on patch in its design which account for its small size and lower cost. The dimensions of slots are $1=20 \mathrm{~mm}$ and $\mathrm{w}=28 \mathrm{~mm}$. the introduction of slot in structure increases the electrical length of the antenna. In third shape a similar h shaped slot has been taken out from the ground plane of the proposed antenna that forms defected ground structure. The dimensions of both the slots of patch and 
ground plane are safe because the dimensions of the patch are limited along its length and its weight the em field at the patch edges undergo fringing. the amount of fringing is function of the dimension of the patch the thickness of the substrate and its dielectric constant $\mathrm{Cr}$. Because of fringing affects the part of the microstrip antenna looks electrically greater than its physical size. for better resonance performance parameters good Impedance Matching is required and it can be calculated using equation 2 and 3

$$
\begin{aligned}
& \varepsilon_{e f f}=\frac{\varepsilon_{r}+1}{2}+\frac{\varepsilon_{r}-1}{2}\left(\frac{1}{\sqrt{1+12 \frac{h}{w}}}\right) \\
& Z_{0}=\frac{120 \Pi}{\sqrt{\varepsilon_{e f f}}\left[\frac{w}{h}+1.393+0.667 \ln \left(\frac{w}{h}+1.444\right)\right]} \text { for } \frac{w}{h} \geq 1
\end{aligned}
$$

\section{RESULTS \& DISCUSSSIONS}

A. Comparison of Resonant performance of microstrip antenna, with H-shaped slot and DGS.

The simulation tool used for evaluating the performance of all proposed antennas is IE3D software. This software is based on the method of the moment's technique. Table. I show the resonant performance characteristics of the planar patch antenna, antenna with slot and with DGS structure. It has been observed that by taking out „, $\mathrm{H}^{\mathrm{ee}}$ shape slot from the patch and from the ground plane, there is considerable improvement in the return loss, VSWR and input impedance. Moreover, the size of the proposed antenna has been reduced to $56.66 \%$ which makes it effectively less expensive comparable to original patch.

Fig. 2 shows the s-parameters of all the proposed small- size planar antennas. From this figure, it is clear that the planar patch antenna and the antenna with slot does not produce any resonant frequency below than -10dB return loss. However, there is a drastic improvement in the resonant properties, especially in terms of return loss of the antenna, when an „, $\mathrm{H}^{\text {ee }}$ shape defect has been introduced in the ground plane of the proposed antenna structure.

\begin{tabular}{|c|c|c|c|c|}
\hline Structure & Resonant Freq. (GHz) & Return Loss (dB) & VSWR & Input Impedance (Ohms) \\
\hline \multirow{5}{*}{ Microstrip Antenna } & 2.07 & -3.41 & 5.13 & 19.41 \\
\hline & 3.89 & -3.38 & 5.20 & 10.20 \\
\hline & 5.47 & -3.89 & 5.01 & 11.99 \\
\hline & 6.99 & -5.28 & 3.38 & 15.81 \\
\hline & 8.52 & -6.12 & 2.96 & 17.19 \\
\hline \multirow{4}{*}{ With Slot } & 1.46 & -2.78 & 6.30 & 57.94 \\
\hline & 5.02 & -6.26 & 2.89 & 53.01 \\
\hline & 6.60 & -3.60 & 4.88 & 29.03 \\
\hline & 8.32 & -4.91 & 3.61 & 13.90 \\
\hline \multirow{6}{*}{ With Slot and DGS } & 1.84 & -15.50 & 1.40 & 46.65 \\
\hline & 5.00 & -17.88 & 1.29 & 53.41 \\
\hline & 5.52 & -14.34 & 1.47 & 37.77 \\
\hline & 6.24 & -16.56 & 1.34 & 51.23 \\
\hline & 7.65 & -14.46 & 1.46 & 39.91 \\
\hline & 8.62 & -12.9 & 1.58 & 31.62 \\
\hline
\end{tabular}

Table. I COMPARISON OF RESONANT PERFORMANCE CHARACTERISTICS. 

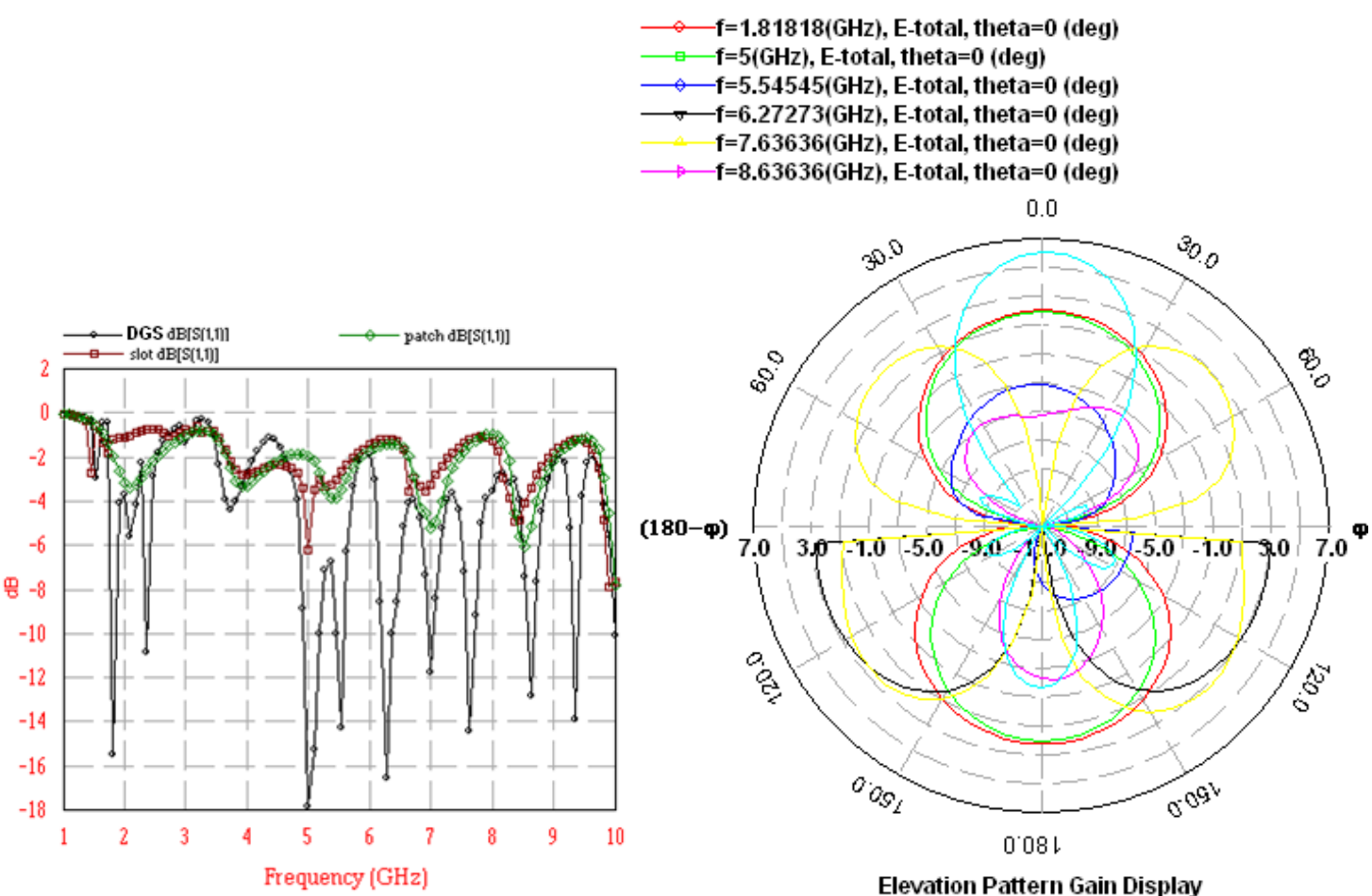

Fig.2 S-parameters of the Proposed Patch, Slot Antenna and DGS

With these resonant properties the proposed antenna can be used for S-band, C-band and X-band wireless applications.

\section{B. Radiation Pattern and Gain.}

The radiation patterns of proposed patch antenna with DGS are shown in Fig.4. It has been observed that they are quite similar in nature for all the frequency bands. The radiation pattern is symmetrical to the antenna axis in E- plane, whereas in H-Plane, it is nearly omni-directional.

The Gain for the proposed antennas is shown in Fig.3. The maximum achievable gain for the antenna is $6.12 \mathrm{dBi}$ at $8.717 \mathrm{GHz}$.

(a)

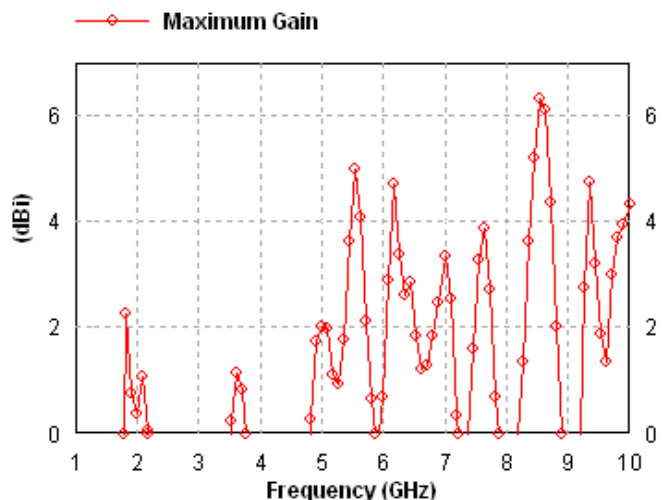

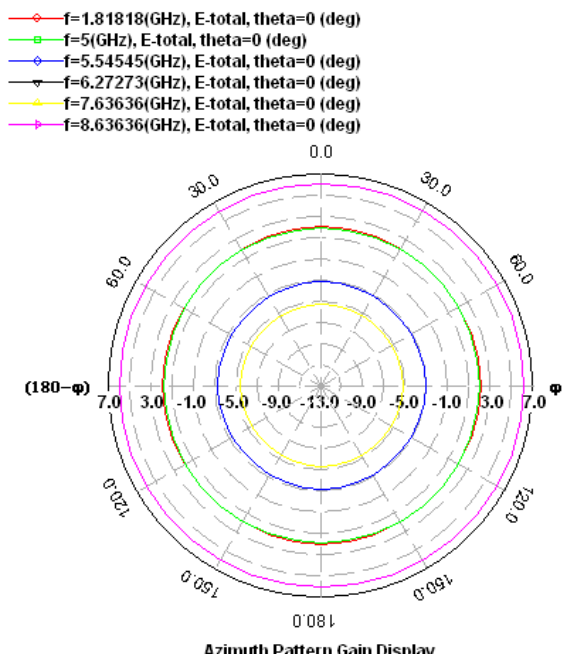

(b)

Azimuth Pattern Gain Displav

Fig.4 Simulated Radiation patterns of proposed antenna with DGS (a) E-plane (b) H-plane

\section{CONCLUSION}

In this paper, the microstrip patch antenna with DGS and miniature sizes are employed. The results are compared with original patch antenna and further with the slot antenna. These results has been revealed that in addition to the reduction of the size of the antenna by $56.66 \%$, there is considerable improvement in return loss, input impedance and VSWR with the introduction of 
„H $\mathrm{H}^{\text {ee }}$ shape defect in ground plane. The simulated results illustrated that the proposed antenna is suitable for S-band, C-band and $\mathrm{X}$-band wireless applications.

\section{REFERENCES}

[1] A. Balanis, "Antenna Theory”. John Wiley \& Sons, Inc. 1997. "SEPE:

[2] M. Hirvoven, P. Pusula, K. Jaakkola and K. Laukkanen, "Planar Inverted F- Antenna for Radio Frequency Identification", isEP Electronic Letters, vol. 40 , no. 14 , pp. $848-850$, 2004.

[3] F.Yong, X. Zhang, X. Ye, and Y. Rahmat-Samii, Wide-band E- shaped patch antennas for wireless communication," IEEE Transactions on Antennas and Propagaion., Vol. 49, No. 7, isEppp.1094-1100, Jul. 2001. isEp?

[4] Jen-Yea Jan and Jia-wei Su, "Bandwidth enhancement of a printed isepe:wide slot antenna with a rotated slot," IEEE Transactions on Antennas and Propagation, Vol.53, No.6, pp.2111-2114, June 2005. ische?

[5] K.Chung, T.Yun and J.Choi, "Wideband CPW-fed monopole antenna with parasitic elements and slots.”Electronics Letters,vol.40,pp.10381040,2004 istep:

[6] J. Jan and J. Su, "Bandwidth enhancement of a printed wide slot antenna with a rotated slot," IEEE Transactions on Antennas andPropagation, Vol.53, No.6, pp.2111-2114, June 2005. "ispep?

[7] L. Weng, Y. Guo, X. Shi and X. Chen, “An Overview of Defected Ground Structure,” Progress In Electromagnetics Research B, vol. 7, pp. 173189,2008 . SSEP

[8] J. Geng, J. Li, R. Jin, S. Ye, X. Liang and M. Li, "The Development of Curved Microstrip Antenna with Defected Ground Structure," Progress In Electromagnetics Research, PIER, vol. 98, pp. 53-73, 2009 sispep? 